\section{Camões M}

Lopes C

\section{Fatores associados à atividade física na população portuguesa}

\author{
Factors associated with physical \\ activity in the Portuguese population
}

\section{RESUMO}

OBJETIVO: Avaliar como as características demográficas, sociais e comportamentais se associam a diferentes tipos de atividade física.

MÉTODOS: Avaliaram-se 37.692 indivíduos de amostra representativa da população portuguesa, no âmbito do Inquérito Nacional de Saúde, 1998-99. A maioria era constituída por mulheres $(53,1 \%)$ e idade $\geq 20$ anos. A avaliação da atividade física diária foi baseada em questionário e classificada como: total, de lazer e exercício. Cada tipo foi dicotomizado em baixa intensidade (atividades leves/moderadas) e alta intensidade (atividades pesadas/muito pesadas). Calcularam-se odds ratios (OR) e respectivos intervalos de confiança de $95 \%$ por regressão logística não condicional.

RESULTADOS: Em ambos os sexos, verificou-se associação inversa significativa entre idade e diferentes tipos de atividade física, e entre a obesidade e a atividade de lazer e exercício. A escolaridade $(\leq 4 ; 5-11 ; \geq 12$ anos) associou-se positivamente com a atividade física de lazer (OR 1; 1,58; 2,39 nas mulheres e OR $1 ; 1,44 ; 2,08$ nos homens) e com o exercício (OR 1 ; 3,$50 ; 9,77$ nas mulheres e OR $1 ; 3,42 ; 7,61$ nos homens) e de forma inversa com a AF total (OR 1; 0,65; 0,20 nas mulheres e 1; 0,47; 0,09 nos homens). Independentemente da idade, os solteiros eram frequentemente mais ativos. A atividade de lazer associou-se negativamente ao consumo de bebidas alcoólicas para ambos os sexos e nos homens, ao consumo de tabaco.

CONCLUSÕES: Os jovens, normoponderais, solteiros, não-bebedores e os homens não-fumadores apresentaram maior probabilidade de serem fisicamente ativos. Em ambos os sexos, observou-se um efeito diferencial da escolaridade segundo os tipos de atividade física.

DESCRITORES: Atividade Física. Exercício. Fatores Etários. Fatores Socioeconômicos. Levantamentos Epidemiológicos.
Serviço de Higiene e Epidemiologia. Faculdade de Medicina. Universidade do Porto. Porto, Portugal

Correspondência | Correspondence: Miguel Camões

Serviço de Higiene e Epidemiologia

Faculdade de Medicina da Universidade do

Porto

Alameda Prof. Hernâni Monteiro

4200-319 Porto, Portugal

E-mail: mcamoes@med.up.pt 


\begin{abstract}
OBJECTIVE: To evaluate the association between demographic, social and behavior characteristics and different types of physical activity.

METHODS: A total of 37.692 subjects of a representative sample of the Portuguese population were studied as part of the National Health Survey in 1998 and 1999. Most were females (53.1\%) aged $\geq 20$ years. Daily physical activity was self-reported based on a questionnaire and discriminated in different types: total physical activity, leisure-time and exercise. Each type of physical activity was dichotomized into low (light/moderate) and high intensity (heavy/very heavy). Odds ratios (OR) and 95\% confidence intervals were estimated using unconditional logistic regression.
\end{abstract}

RESULTS: In both men and women, a significant inverse association between age and different types of physical activity and between obesity and leisure time physical activity and exercise was seen. A positive association was found between education $(\leq 4 ; 5-11 ; \geq 12$ years) and leisure-time PA (OR $1 ; 1.58$; 2.39 in females and $1 ; 1.44 ; 2.08$ in males) and exercise (OR $1 ; 3.50 ; 9.77$ in females and $1 ; 3.42 ; 7.61$ in males) and an inverse association with total physical activity (OR $1 ; 0.65 ; 0.20$ in females and $1 ; 0.47 ; 0.09$ in males) was found. Regardless of age, single subjects were frequently more active. Alcohol consumption in both men and women and tobacco consumption in males were negatively associated with leisure-time physical activity.

CONCLUSIONS: Youngest, normal weighted, single, non-drinkers subjects and non-smokers males were more likely to be physically active. In both men and women, there was a clear effect of education on the type of physical activity.

DESCRIPTORS: Physical Activity. Exercise. Age Factors. Socioeconomic Factors. Health Surveys.

\title{
INTRODUÇÃO
}

A atividade física regular é determinante importante na redução do risco de doença cardiovascular e apresenta impacto significativo em todas as causas de mortalidade. ${ }^{6}$ Outros benefícios surgem em nível psicossocial, como a redução do stress e da sintomatologia depressiva e o aumento da sensação de bem-estar, envolvendo maiores níveis de autoconfiança e conseqüente satisfação pessoal. ${ }^{4,18}$

Apesar de os benefícios da atividade física na saúde se encontrarem bem documentados, ${ }^{6}$ o comportamento sedentário é característico da civilização moderna. ${ }^{20} \mathrm{No}$ decorrer dos últimos 50 anos, a atividade física ocupacional teve decréscimo acentuado, ${ }^{2}$ pois a mecanização do trabalho e das atividades domésticas diminuiu de forma significativa a atividade física da vida moderna. Assim, a atividade física de lazer assumiu papel relevante no cumprimento das necessidades de atividade física diária. ${ }^{19}$

Em 1997, entre 15 países da União Européia, ${ }^{20}$ a população portuguesa era a que apresentava prevalência mais alta de sedentarismo (87,8\%). Com base na mesma amostra Européia, ${ }^{21}$ foi descrito que a participação em qualquer tipo de atividade física decresce significativamente com o aumento do IMC. A par desta situação encontra-se a elevada prevalência de excesso de peso e obesidade na população portuguesa. ${ }^{13}$

A atividade física é definida como qualquer movimento corporal produzido pelo músculo esquelético que resulta num aumento do dispêndio energético, ${ }^{3}$ constituindo-se processo complexo e dinâmico. Durante a vida, o indivíduo passa por fases que evidenciam diferentes níveis de atividade física, determinados por diversos fatores. Como determinantes desse complexo comportamento, duas categorias podem influenciar os padrões de atividade física: as características individuais, incluindo motivações, auto-eficácia, habilidades motoras e outros comportamentos de saúde; e as características ambientais, como o acesso ao trabalho ou espaços de lazer, custos, barreiras de disponibilidade temporal e suporte sociocultural. ${ }^{19}$

Características sociodemográficas como sexo, idade, escolaridade, ocupação e estado civil parecem assumirse como fatores determinantes de um estilo de vida sedentário. ${ }^{5} \mathrm{O}$ consumo de tabaco e de álcool são alguns 
dos fatores comportamentais, que se encontram também associados à atividade física. Maiores níveis de atividade física reduzem o risco de fumar durante a adolescência ${ }^{1}$ e o consumo moderado de álcool está associado a estilos de vida saudáveis. ${ }^{15}$ Contudo, as diferenças na associação entre esses determinantes e os diferentes tipos de atividade física, permanecem por esclarecer.

O presente estudo teve por objetivo avaliar como as características sociais, demográficas e comportamentais se associam aos diferentes tipos de atividade física.

\section{MÉTODOS}

Foram utilizados dados do Inquérito Nacional de Saúde, conduzido entre outubro de 1998 e setembro de 1999. a A amostra de base foi probabilística de áreas e multietápica, construída a partir dos dados do Recenseamento da População e Habitação de 1991 e que considera as unidades de alojamento. A população abrangida foi constituída pelo conjunto de indivíduos que residem em alojamentos familiares, selecionada a partir da amostra de base, representativa das principais regiões de Portugal Continental (Norte, Centro, Lisboa, Alentejo e Algarve).

A informação foi coletada por inquiridores treinados de acordo com o protocolo padronizado, submetida a controle de qualidade. Foi obtida proporção de participação de $82 \%$. De 48.606 indivíduos encontrados, foram excluídos aqueles com idade $<20$ anos e os que possuíam incapacidade de longa duração, perfazendo 37.692 sujeitos (53,1\% mulheres e $46,9 \%$ homens).

Os dados de identificação, caracterização social e comportamental foram auto-declarados: tais como a idade, escolaridade, peso, estatura, estado civil, consumo de tabaco e de álcool. A avaliação da atividade física diária foi baseada nas seguintes questões: 1) Qual das situações descreve melhor o esforço exigido pela atividade diária? (exemplos de atividades leves/moderadas/pesadas/muito pesadas); 2) Qual das situações descreve melhor as atividades de tempo livre? (exemplos de atividades leves/moderadas/pesadas/muito pesadas); 3) Pelo menos uma vez por semana, dedica-se a alguma atividade física regular (correr, andar de bicicleta ou outras) o suficiente para começar a sentir cansaço? ( $\mathrm{sim} /$ não). A cada questão fez-se corresponder um tipo de atividade física: atividade física total, atividade física de lazer e exercício físico, sendo cada variável dicotomizada em baixa intensidade (atividades leves/moderadas) e alta intensidade (atividades pesadas/muito pesadas).

Os indivíduos foram categorizados de acordo com classes de idade (anos): 20-34, 35-49, 50-64, $\geq 65$; escolaridade (anos): $\leq 4,5-11, \geq 12$; estado civil: casado, solteiro, viúvo e divorciado e IMC: $<25,25-29,9, \geq 30$.
O consumo de tabaco foi classificado em: fumadores (regulares - consumo diário; ocasionais - menos de uma vez por dia) e não-fumadores (não-fumadores atuais). O consumo de bebidas alcoólicas referente aos 12 meses anteriores à entrevista foi categorizado em: bebedores regulares (pelo menos uma vez por mês) e não-bebedores (não-bebedores e bebedores esporádicos - menos de uma vez por mês).

Para comparação de proporções dos indivíduos com baixa e alta intensidade de atividade física por categoria de cada característica estudada utilizou-se o teste qui-quadrado.

Calcularam-se odds ratios (OR) e intervalos de confiança a 95\% (IC 95\%) por regressão logística não condicional e construíram-se modelos separados para homens e mulheres. Como variável dependente usouse a atividade física, dicotomizada em níveis baixos de atividade (leve/moderada) e em níveis altos de atividade (pesada/muito pesada) para a atividade física total, atividade física de lazer e exercício ( $\operatorname{sim} /$ não). Após análise univariada da associação entre as características demográficas, sociais, comportamentais e tipos de atividade física, construíram-se modelos multivariados. Nestes modelos, a idade, a escolaridade, o IMC, o estado civil e os consumos de tabaco e de álcool entraram simultaneamente como variáveis independentes.

A análise estatística foi feita segundo sexo e utilizando o programa SPSS versão 13.0.

\section{RESULTADOS}

A Tabela 1 mostra a percentagem de mulheres com atividade física de baixa intensidade total $(87,6 \%)$ e de lazer (80,8\%), e 95,5\% não realizavam exercício físico regular. Os homens, apesar de mais ativos, apresentam igualmente elevadas percentagens de níveis baixos de atividade física total $(71,6 \%)$ e de lazer $(68,4 \%)$ e $90,2 \%$ não realizavam exercício físico.

Em ambos os sexos, a proporção de indivíduos com atividade física de lazer de alta intensidade e que realizam exercício físico decresce linearmente segundo as classes de idade e de IMC. A proporção de mulheres que realizam atividades de lazer de alta intensidade e que realizam exercício físico aumenta linearmente com os anos de escolaridade $(\leq 4 ; 5-11 ; \geq 12), 15,5 \% ; 24,4 \%$; $34,5 \%$ na atividade física de lazer e $1,5 \% ; 6,0 \% ; 16,4 \%$ na realização de exercício físico. Não se verifica este tipo de linearidade quando analisada a atividade física total segundo as classes de escolaridade, invertendose o sentido na distribuição das proporções, $14,9 \%$; $13,3 \% ; 6,2 \%$. A proporção de mulheres solteiras $(28,4 \%)$ e divorciadas $(22,3 \%)$ com elevado nível de atividade física de lazer foi superior à proporção de

a Observatório Nacional de Saúde. Instituto Nacional de Saúde Dr. Ricardo Jorge. Inquérito Nacional de Saúde 1998/1999. Lisboa; 2000. [acesso em 23/11/2006]. Disponível em: www.onsa.pt/index_17.html 
Tabela 1. Características sociodemográficas e compotamentais da população estudada segundo tipos de atividade física, por sexo. Portugal, 1998-1999.

\begin{tabular}{|c|c|c|c|c|c|c|}
\hline \multirow{2}{*}{ Variável } & \multicolumn{2}{|c|}{ AF Total N(\%) } & \multicolumn{2}{|c|}{ AF Lazer N(\%) } & \multicolumn{2}{|c|}{ Exercício físico N(\%) } \\
\hline & Baixa intensidade & Alta intensidade & Baixa intensidade & Alta intensidade & Não & Sim \\
\hline \multicolumn{7}{|c|}{ Mulheres } \\
\hline $\mathrm{N}(\%)$ & $17.139(87,6)$ & $2.425(12,4)$ & $15.811(80,8)$ & $3.750(19,2)$ & $18.690(95,5)$ & $874(4,5)$ \\
\hline \multicolumn{7}{|l|}{ Idade (anos) } \\
\hline $20-34$ & $3.977(88,4)$ & $522(11,6)$ & $3.260(72,5)$ & $1.239(27,5)$ & $4.065(90,4)$ & $434(9,6)$ \\
\hline $35-49$ & $4.149(82,1)$ & $905(17,9)$ & $3.930(77,8)$ & $1.124(22,2)$ & $4.789(94,8)$ & $265(5,2)$ \\
\hline $50-64$ & $4.260(85,3)$ & $735(14,7)$ & $4.121(82,5)$ & $872(17,5)$ & $4.868(97,5)$ & $127(2,5)$ \\
\hline$\geq 65$ & $4.753(94,8)$ & $263(5,2)$ & $4.500(89,7)$ & $515(10,3)$ & $4.968(99,0)$ & $48(1,0)$ \\
\hline \multicolumn{7}{|l|}{ Escolaridade (anos) } \\
\hline$\leq 4$ & $7.770(85,1)$ & $1.361(14,9)$ & $7.717(84,5)$ & $1.411(15,5)$ & $8.992(98,5)$ & $138(1,5)$ \\
\hline $5-11$ & $4.008(86,7)$ & $613(13,3)$ & $3.494(75,6)$ & $1.127(24,4)$ & $4.342(94,0)$ & $279(6,0)$ \\
\hline$\geq 12$ & $2.530(93,8)$ & $168(6,2)$ & $1.767(65,5)$ & $932(34,5)$ & $2.257(83,6)$ & $442(16,4)$ \\
\hline \multicolumn{7}{|l|}{ Estado civil } \\
\hline Casado & $11.098(85.4)$ & $1.892(14,6)$ & $10.586(81,5)$ & $2.401(18,5)$ & $12.539(96,5)$ & $450(3,5)$ \\
\hline Solteiro & $2.782(90.9)$ & $277(9,1)$ & $2.190(71,6)$ & $869(28,4)$ & $2.709(88,6)$ & $350(11,4)$ \\
\hline Viúvo & $2.646(94.1)$ & $166(5,9)$ & $2.489(88,5)$ & $323(11,5)$ & $2.775(98,7)$ & $38(1,3)$ \\
\hline Divorciado & $613(87.2)$ & $90(12,8)$ & $546(77,7)$ & $157(22,3)$ & $667(94,9)$ & $36(5,1)$ \\
\hline \multicolumn{7}{|l|}{$\mathrm{IMC}\left(\mathrm{kg} / \mathrm{m}^{2}\right)$} \\
\hline$<25$ & $8.799(88,2)$ & $1.178(11,8)$ & $7.750(77,7)$ & $2.226(22,3)$ & $9.347(93,7)$ & $631(6,3)$ \\
\hline $25-29,9$ & $5.400(86,6)$ & $832(13,4)$ & $5.137(82,4)$ & $1.094(17,6)$ & $6.054(97,2)$ & $177(2,8)$ \\
\hline$\geq 30$ & $2.578(87,6)$ & $366(12,4)$ & $2.572(87,4)$ & $371(12,6)$ & $2.890(98,2)$ & $54(1,8)$ \\
\hline \multicolumn{7}{|l|}{ Tabaco } \\
\hline Fumadores & $1.700(87,4)$ & $244(12,6)^{*}$ & $1.429(73,5)$ & $514(26,5)$ & $1.781(91,6)$ & $163(8,4)$ \\
\hline Não-fumadores & $15.439(87,6)$ & $2.181(12,4)$ & $14.382(81,6)$ & $3.236(18,4)$ & $16.909(96,0)$ & $711(4,0)$ \\
\hline \multicolumn{7}{|l|}{ Álcool } \\
\hline Bebedores & $4.577(85,0)$ & $807(15,0)$ & $4.152(77,1)$ & $1.232(22,9)$ & $5.138(95,4)$ & $247(4,6)^{*}$ \\
\hline Não-bebedores & $12.662(88,6)$ & $1.618(11,4)$ & $11.659(82,2)$ & $2.518(17,8)$ & $13.552(95,6)$ & $627(4,4)$ \\
\hline \multicolumn{7}{|c|}{ Homens } \\
\hline$N(\%)$ & $12.467(71,6)$ & $4.947(28,4)$ & $11.913(68,4)$ & $5.502(31,6)$ & $15.720(90,2)$ & $1.712(9,8)$ \\
\hline \multicolumn{7}{|l|}{ Idade (anos) } \\
\hline $20-34$ & $2.971(63,8)$ & $1.686(36,2)$ & $2.753(59,1)$ & $1.907(40,9)$ & $3.647(78,3)$ & $1.014(21,7)$ \\
\hline $35-49$ & $2.950(64,5)$ & $1.627(35,5)$ & $3.106(67,9)$ & $1.471(32,1)$ & $4.127(90,2)$ & $450(9,8)$ \\
\hline $50-64$ & $3.129(72,3)$ & $1.201(27,7)$ & $3.045(70,3)$ & $1.284(29,7)$ & $4.146(95,7)$ & $183(4,3)$ \\
\hline$\geq 65$ & $3.417(88,8)$ & $433(11,2)$ & $3.009(78,2)$ & $840(21,8)$ & $3.785(98,3)$ & $65(1,7)$ \\
\hline \multicolumn{7}{|l|}{ Escolaridade (anos) } \\
\hline$\leq 4$ & $5.440(67,1)$ & $2.670(32,9)$ & $5.968(73,6)$ & $2.142(26,4)$ & $7.864(97,0)$ & $247(3,0)$ \\
\hline $5-11$ & $3.758(68,4)$ & $1.736(31,6)$ & $3.468(63,1)$ & $2.025(36,9)$ & $4.682(85,2)$ & $812(14,8)$ \\
\hline$\geq 12$ & $1.979(91,0)$ & $196(9,0)$ & $1.149(52,8)$ & $1.028(47,2)$ & $1.543(70,9)$ & $634(29,1)$ \\
\hline \multicolumn{7}{|l|}{ Estado civil } \\
\hline Casado & $9.082(71,4)$ & $3.637(28,6)$ & $8.994(70,7)$ & $3.724(29,3)$ & $11.839(93,1)$ & $880(6,9)$ \\
\hline Solteiro & $2.594(69,2)$ & $1.153(30,8)$ & $2.214(59,1)$ & $1.535(40,9)$ & $2.958(78,9)$ & $792(21,1)$ \\
\hline Viúvo & $549(89,4)$ & $65(10,6)$ & $474(77,2)$ & $140(22,8)$ & $603(98,2)$ & $11(1,8)$ \\
\hline Divorciado & $242(72,5)$ & $92(27,5)$ & $231(69,2)$ & $103(30,8)$ & $305(91,3)$ & $29(8,7)$ \\
\hline \multicolumn{7}{|l|}{$\mathrm{IMC}\left(\mathrm{kg} / \mathrm{m}^{2}\right)$} \\
\hline$<25$ & $5.325(71,7)$ & $2.099(28,3)^{*}$ & $4.867(65,5)$ & $2.559(34,5)$ & $6.494(87,5)$ & $933(12.5)$ \\
\hline $25-29,9$ & $5.278(71,0)$ & $2.158(29,0)$ & $5.122(68,9)$ & $2.313(31,1)$ & $6.112(91,6)$ & $624(8,4)$ \\
\hline$\geq 30$ & $1.674(73,3)$ & $609(26,7)$ & $1.743(76,3)$ & $540(23,7)$ & $2.164(94,8)$ & $119(5,2)$ \\
\hline \multicolumn{7}{|l|}{ Tabaco } \\
\hline Fumadores & $4.098(67,5)$ & $1.969(32,5)$ & $4.253(70,1)$ & $1.814(29,9)$ & $5.433(89,6)$ & $634(10,4)$ \\
\hline Não-fumadores & $8.369(73,8)$ & $2.978(26,2)$ & $7.660(67,5)$ & $3.688(32,5)$ & $10.272(90,5)$ & $1.078(9,5)$ \\
\hline \multicolumn{7}{|l|}{ Álcool } \\
\hline Bebedores & $8.536(68,3)$ & $3.963(31,7)$ & $8.539(68,3)$ & $3.960(31,7)^{*}$ & $11.401(91,2)$ & $1.099(8,8)$ \\
\hline Não-bebedores & $3.931(80,0)$ & $984(20,0)$ & $3.374(68,6)$ & $1.542(31,4)$ & $4.304(87,5)$ & $613(12,5)$ \\
\hline
\end{tabular}

AF: atividade física

$* \mathrm{p}<0,05$ 
mulheres casadas $(18,5 \%)$ e viúvas $(11,5 \%)$. Observouse a mesma distribuição de proporções nas mulheres que realizavam exercício físico regular, com maior proporção entre as solteiras $(11,4 \%)$ e divorciadas $(5,1 \%)$. Entre mulheres consumidoras de tabaco ou de álcool observou-se uma maior proporção de mulheres envolvidas em atividades de alta intensidade, para todos os tipos de atividade física quando comparadas com as não consumidoras (Tabela 1).

Os homens mais escolarizados ( $\geq 12$ anos) eram os que estavam envolvidos em atividades de lazer de maior intensidade e na prática de exercício físico, em comparação com os que possuíam menos escolaridade $(\leq 4$ anos): respectivamente, $47,2 \%$ vs. $26,4 \%$ na atividade de lazer, e $29,1 \%$ vs. 3,0\% no exercício físico. A linearidade da distribuição das proporções por categoria de escolaridade $(\leq 4 ; 5-11 ; \geq 12)$ inverte o sentido quando analisada a atividade física total de alta intensidade, $32,9 \% ; 31,6 \% ; 9,0 \%$.

Os homens solteiros apresentaram comportamento semelhante às mulheres, nos quais se observou maior proporção de indivíduos com maior intensidade de atividade, em todos os tipos de atividade física. Os homens não-fumadores mostraram-se ser mais ativos no lazer que os fumadores $(32,5 \%$ vs. $29,9 \%$, $\mathrm{p}<0,001)$ assim como a proporção de homens não-bebedores que realizam exercício físico foi maior que os bebedores envolvidos na mesma atividade física $(12,5 \%$ vs. $8,8 \%, \mathrm{p}<0,001)$.

Na Tabela 2 encontra-se descrita a associação entre as características sociodemográficas e comportamentais e o nível de atividade física dos indivíduos, tendo em consideração os tipos de atividade. Em ambos os sexos observou-se, em análise univariada, associação inversa entre a idade e maiores níveis de atividade total, de lazer e fazer exercício físico. Após ajuste para as demais características, que em bruto se associavam significativamente com a atividade física, a associação inversa significativa manteve-se nas mulheres com idade $\geq 65$ anos e nos homens em todas as classes de idade levando em consideração a atividade física total $(p<0,001)$, a de lazer $(\mathrm{p}<0,001)$ e o exercício físico $(\mathrm{p}<0,001)$, tendo como classe de referência os indivíduos mais novos (20-34 anos) (Tabela 2).

A escolaridade associou-se de forma distinta em relação aos tipos de atividade física, em ambos os sexos. Em análise multivariada, indivíduos com maior escolaridade ( $\geq 12$ anos) apresentavam menor probabilidade de ter atividade diária de alta intensidade, considerando como classe de referência os menos escolarizados $(\leq 4$ anos). Mulheres e homens com maior escolaridade possuíam probabilidade duas vezes maior de serem fisicamente ativos no lazer que os menos escolarizados e a associação aumenta de quando se analisa a prática de exercício físico.
Os indivíduos solteiros tinham maior probabilidade de terem altos níveis de atividade física de lazer e de realizarem exercício físico regular, tendo como classe de referência os indivíduos casados. Quando analisada a atividade física total, as mulheres solteiras apresentaram menor probabilidade de serem fisicamente ativas.

Em análise univariada, encontrou-se em ambos os sexos associação inversa significativa entre o IMC e ser mais ativo nos tipos de atividade física. Essa associação manteve-se após ajuste e em ambos os sexos, mostrando-se consistente na categoria de obesidade $\left(\geq 30 \mathrm{~kg} / \mathrm{m}^{2}\right) \mathrm{em}$ comparação com os indivíduos com IMC $<25 \mathrm{~kg} / \mathrm{m}^{2}$, tanto na atividade física de lazer como no exercício físico (Tabela 2).

Em análise multivariada relativa a hábitos, as mulheres não-bebedoras tinham maior probabilidade de serem mais ativas nos diferentes tipos de atividade em relação às bebedoras na atividade física total, de lazer e na prática de exercício físico. Os homens que não consumiam álcool tinham maior probabilidade de serem mais ativos tanto na atividade total como na de lazer. Os homens não-fumadores tinham maior probabilidade de serem mais ativos nos tempos de lazer e de fazerem exercício físico que os não-fumadores. As mulheres não-fumadoras tinham menor probabilidade de terem atividade física de lazer de elevada intensidade e de realizarem exercício físico, mas essa associação perdeu significado estatístico após ajuste.

\section{DISCUSSÃO}

Os resultados do presente trabalho mostraram que a prevalência de atividade física de elevada intensidade e da prática de exercício físico variou de acordo com as características demográficas, sociais, e comportamentais. Idade, escolaridade, estado civil, IMC e hábitos tabágicos e alcoólicos dos indivíduos associam-se de forma independente com a atividade física, com efeito diferencial da escolaridade de acordo com os tipos de atividade física.

A proporção de indivíduos com baixos níveis de atividade física é elevada na população portuguesa. De acordo com os tipos de atividade, a proporção de mulheres com atividade física de baixa intensidade variou entre os $80,8 \%$ da atividade física de lazer e os $95,5 \%$ da não participação na prática de exercício físico. Nos homens, o padrão de inatividade é pouco mais favorável, com $68,4 \%$ deles apresentarem baixos níveis de intensidade de atividade física de lazer e $90,2 \%$ a não realizarem qualquer tipo de exercício físico, sendo no entanto, em percentagem inferior ao descrito em alguns países europeus. ${ }^{20}$ Nesse estudo, ${ }^{20}$ a prevalência de sedentarismo encontrada em Portugal foi de $87,8 \%$ na atividade física de lazer. Entre 15 países da União Européia estudados, aqueles ao norte da Europa como a Suécia (43,3\%), a Irlanda $(44,1 \%)$, a Finlândia $(48,6 \%)$ e a Áustria $(46,8 \%)$ na Europa 
Tabela 2. Associação entre variáveis sociodemográficas, comportamentais e tipos de atividade física, por sexo. Portugal, 1998-1999.

\begin{tabular}{|c|c|c|c|c|c|c|}
\hline \multirow[b]{2}{*}{ Variável } & \multicolumn{2}{|c|}{ AF Total } & \multicolumn{2}{|c|}{ AF Lazer } & \multicolumn{2}{|c|}{ Exercício físico } \\
\hline & $\begin{array}{l}\text { OR Bruto } \\
\text { (IC 95\%) }\end{array}$ & $\begin{array}{l}\text { OR Ajustado* } \\
\text { (IC 95\%) }\end{array}$ & $\begin{array}{l}\text { OR Bruto } \\
\text { (IC 95\%) }\end{array}$ & $\begin{array}{l}\text { OR Ajustado* } \\
\text { (IC 95\%) }\end{array}$ & $\begin{array}{l}\text { OR Bruto } \\
\text { (IC 95\%) }\end{array}$ & $\begin{array}{l}\text { OR Ajustado* } \\
\text { (IC } 95 \%)\end{array}$ \\
\hline \multicolumn{7}{|c|}{ Mulheres } \\
\hline \multicolumn{7}{|c|}{ Idade (anos) } \\
\hline $20-34$ & 1 & 1 & 1 & 1 & 1 & 1 \\
\hline $35-49$ & $1,66(1,48 ; 1,86)$ & $1,12(0,98 ; 1,29)$ & $0,75(0,68 ; 0,82)$ & $1,05(0,94 ; 1,17)$ & $0,51(0,44 ; 0,60)$ & $1,19(0,98 ; 1,44)$ \\
\hline $50-64$ & $1,31(1,16 ; 1,48)$ & $0,74(0,64 ; 0,87)$ & $0,55(0,50 ; 0,61)$ & $1,01(0,89 ; 1,14)$ & $0,24(0,20 ; 0,29)$ & $1,06(0,82 ; 1,36)$ \\
\hline$\geq 65$ & $0,42(0,36 ; 0.39)$ & $0,26(0,20 ; 0,32)$ & $0,30(0,26 ; 0,33)$ & $0,65(0,55 ; 0,76)$ & $0,09(0,06 ; 0,12)$ & $0,53(0,35 ; 0,79)$ \\
\hline
\end{tabular}

Escolaridade (anos)

$\begin{array}{lcccccc}\leq 4 & 1 & 1 & 1 & 1 & 1 & 1 \\ 5-11 & 0,87(0,78 ; 0,96) & 0,65(0,57 ; 0,73) & 1,76(1,61 ; 1,92) & 1,58(1,42 ; 1,75) & 4,18(3,40 ; 5,15) & 3,50(2,77 ; 4,42) \\ \geq 12 & 0,37(0,32 ; 0,44) & 0,29(0,24 ; 0,35) & 2,88(2,61 ; 3,18) & 2,39(2,12 ; 2,70) & 12,76(10,48 ; 15,53) & 9,77(7,70 ; 12,40)\end{array}$

Estado civil

Casado

Solteiro

Viúvo

Divorciado

IMC $\left(\mathrm{kg} / \mathrm{m}^{2}\right)$

$<25$

$25-29,9$

$\geq 30$

Tabaco

Fumadores

Não-

fumadores

Álcool

Bebedores

Não-

bebedores

$\begin{array}{llll}0,58(0,51 ; 0,66) & 0,72(0,61 ; 0,85) & 1,75(1,59 ; 1,91) & 1,21(1,08 ; 1,35) \\ 0,36(0,31 ; 0,43) & 0,63(0,51 ; 0,78) & 0,57(0,50 ; 0,64) & 0,90(0,76 ; 1,05) \\ 0,86(0,68 ; 1,08) & 0,84(0,65 ; 1,08) & 1,26(1,05 ; 1,52) & 1,13(0,93 ; 1,38)\end{array}$

1

1

1

$1,15(1,04 ; 1,26) \quad 1,06(0,95 ; 1,18) \quad 0,74(0,68 ; 0,80) \quad 0,94(0,86 ; 1,03)$

$1,06(0,93 ; 1,20) \quad 1,04(0,90 ; 1,19) \quad 0,50(0,44 ; 0,56) \quad 0,71(0,62 ; 0,81)$

1

1

$0,98(0,85 ; 1,13) \quad 0,96(0,82 ; 1,12) \quad 0,62(0,56 ; 0,69) \quad 1,08(0,96 ; 1,21)$

$0,45(0,38 ; 0,54)$

$1,15(0,95 ; 1,39)$

1

$1,36(1,25 ; 1,49) \quad 1,18(1,06 ; 1,30) \quad 1,37(1,27 ; 1,48) \quad 1,37(1,26 ; 1,49)$

$1,03(0,89 ; 1,20)$

$1,21(1,02 ; 1,42)$

Homens

Idade (anos)

20-34

$35-49$

50-64

$\geq 65$

Escolaridade (anos)

$\leq 4$

5-11

$\geq 12$

Estado civil

Casado

Solteiro

Viúvo

Divorciado

IMC $\left(\mathrm{kg} / \mathrm{m}^{2}\right)$

$<25$

$25-29,9$

$\geq 30$

Tabaco

Fumadores

Não-

fumadores

Álcool

Bebedores

Não-

bebedores

$\begin{array}{cccc}1 & 1 & 1 & 1 \\ 0,97(0,89 ; 1,05) & 0,55(0,49,0,61) & 0,68(0,62 ; 0,74) & 0,88(0,79 ; 0,98) \\ 0,67(0,61 ; 0,74) & 0,27(0,24 ; 0,31) & 0,60(0,55 ; 0,66) & 0,88(0,78 ; 0,99) \\ 0,22(0,19 ; 0,25) & 0,09(0,07 ; 0,10) & 0,40(0,36 ; 0,44) & 0,64(0,56 ; 0,73)\end{array}$

$0,22(0,19 ; 0,25) \quad 0,09(0,07 ; 0,10) \quad 0,40(0,36 ; 0,44) \quad 0,64(0,56 ; 0,73)$

$3,60(3,11 ; 4,16)$

$0,38(0,27 ; 0,53)$

$1,52(1,27 ; 1,83)$

$1,06(0,71 ; 1,79)$

$1,08(0,75 ; 1,76)$

$0,43(0,36 ; 0,51)$

1

$0,82(0,68 ; 0,99)$

$0,65(0,48 ; 0,89)$

\title{
1
}

(1,

$1,50(1,06 ; 2,13)$

\author{
(
}

\section{1}

$0,39(0,34 ; 0,44)$

$0,15(0,13 ; 0,18)$

$0.06(0,04 ; 0,08)$

1

$0,68(0,59 ; 0,80)$

$0,41(0,33 ; 0,50)$

$0,18(0,13 ; 0,24)$

\section{1} $0,94(0,87 ; 1,01) \quad 0,47(0,43 ; 0,51) \quad 1,62(1,51 ; 1,75) \quad 1,44(1,32 ; 1,57) \quad 5,52(4,76 ; 6,39) \quad 3,42(2,91 ; 4,03)$ $0,20(0,17 ; 0,23) \quad 0,09(0,08 ; 0,11) \quad 2,49(2,26 ; 2,74) \quad 2,08(1,86 ; 2,32) \quad 13,08(11,18 ; 15,30) \quad 7,61(6,38 ; 9,07)$

* Odds ratios ajustado para todas as variáveis na tabela. 
central são os que apresentam menores percentagens de sedentarismo durante o lazer. Esses resultados vêm dar continuidade às elevadas prevalências de inatividade descritas na mesma amostra européia onde Portugal se destacava pela elevada proporção de inatividade: $60 \%$ dos adultos não realizavam qualquer tipo de atividade de lazer e $80 \%$ não estavam envolvidos em prática desportiva. ${ }^{21}$ Apesar das limitações na comparação de resultados devido às diferentes definições de sedentarismo utilizadas, encontra-se descrito, na população do Porto, ${ }^{8}$ prevalências semelhantes às encontradas na presente análise. Nos Estados Unidos, ${ }^{12}$ mais de metade dos adultos não são regularmente ativos, no entanto, a proporção de adultos inativos nos tempos de lazer decresceu entre 1994 (29,8\%) e 2004 (23,7\%). Essas proporções são semelhantes às prevalências encontradas no presente estudo, mas no que concerne aos indivíduos envolvidos em atividades de lazer de alta intensidade (19,2\% nas mulheres e 31,6\% nos homens).

Num estudo descritivo sobre padrões de vida, ${ }^{14}$ realizado no Brasil em 1996 e 1997, apenas 13\% dos inquiridos reportaram realizar pelo menos $30 \mathrm{~min}$ de atividade física de lazer em um ou mais dias da semana, observando-se uma percentagem muito baixa $(3,3 \%)$ de indivíduos que realizam a mesma duração de atividade física de lazer, cinco ou mais vezes por semana.

Em relação ao aumento da probabilidade de sedentarismo de acordo com a idade nos tempos de lazer, resultados semelhantes foram encontrados na população do Porto. ${ }^{8} \mathrm{O}$ declínio da atividade física com a idade é uma observação consistente na epidemiologia da atividade física, existindo evidência de decréscimo acentuado do dispêndio energético entre a adolescência e a vida adulta. ${ }^{17}$ Com o aumento da idade, diminui a exigência física, principalmente em termos profissionais. No entanto, a relação inversa que existe entre as atividades profissionais e a idade deveria ser compensada por aumento de atividade física de lazer e exercício físico, o que habitualmente não se observa.

Em ambos os sexos, observou-se uma associação significativa e positiva entre o aumento da escolaridade e a atividade física de lazer, sendo de maior magnitude o efeito independente dos anos de escolaridade na prática de exercício físico regular. Quando analisamos o nível de escolaridade com a atividade física total, o sentido da associação inverte. Esse tipo de associação sugere que os portugueses ao reportarem o esforço que melhor caracteriza o seu dia-a-dia tendem a reflectir essencialmente a sua atividade profissional, tornandose fundamental fazer uma distinção clara da atividade física profissional/ocupacional da atividade física de lazer e mesmo do exercício físico na avaliação dos seus determinantes. Os resultados obtidos vão de encontro aos resultados descritos numa população urbana portuguesa, ${ }^{8}$ no qual existia maior probabilidade de um indivíduo ser sedentário na atividade física total com o aumento dos anos de escolaridade. Também outras análises em amostras da população portuguesa ${ }^{20}$ observaram que o nível de escolaridade é determinante da atividade física, independente de fatores que influenciam diretamente este complexo comportamento. Os indivíduos com o nível primário de escolaridade têm $50 \%$ mais probabilidade de serem sedentários na vida adulta, no decorrer dos tempos de lazer, do que os indivíduos que completaram o terceiro ciclo (12 anos) e os que finalizaram o ensino superior. ${ }^{20}$

Outro estudo, tendo como base o Inquérito Nacional de Saúde Canadense e utilizando uma larga amostra de idosos ( $\geq 65$ anos), ${ }^{11}$ descreve associação significativa positiva entre a escolaridade e a atividade física de lazer. Estudo transversal com adultos norte americanos ${ }^{9}$ mostrou o efeito da escolaridade nos tipos de atividade física em análise multivariada, evidenciando tendência linear positiva entre anos de escolaridade e atividade física de lazer. Em contraste, associações inversas com os anos de escolaridade foram encontradas em ambos os sexos ao estudar as atividades físicas profissional, domésticas e total. ${ }^{9}$

O estado civil relaciona-se igualmente com o nível de atividade física e, apesar de pouco descrito, encontra-se na literatura que os indivíduos solteiros são os mais ativos, ${ }^{19,20}$ resultados semelhantes ao do presente estudo. Numa amostra de 15 estados membros da União Européia, ${ }^{20}$ foi descrita maior probabilidade de comportamento sedentário entre indivíduos viúvos e divorciados em comparação com os indivíduos solteiros, sustentando a evidência encontrada na presente análise. Em estudo canadense, ${ }^{11}$ os indivíduos casados, mesmo em idade avançada, possuíam menor probabilidade de serem ativos do que os solteiros.

$\mathrm{O}$ fato de indivíduos com IMC mais elevado apresentaram menor probabilidade de serem mais ativos na atividade física de lazer e na prática de exercício já foi descrito em outros estudos na população portuguesa ${ }^{8,20}$ Dois estudos que avaliaram transversalmente esta relação, ${ }^{11,16}$ relatam declínio da atividade física de lazer associado ao aumento do IMC. Apesar da limitação do desenho de estudos transversais, indivíduos com maior IMC são menos ativos, realçando a importância da promoção da atividade física sob a perspectiva de prevenção primária, isto é, antes do excesso de peso e a obesidade se instalar.

A associação entre o consumo de tabaco e o comportamento sedentário foi descrita no estudo europeu. ${ }^{20}$ Indivíduos fumadores apresentaram cerca de 50\% mais risco de serem sedentários nos tempos de lazer, que os não-fumadores, embora tenha sido avaliada a distribuição dos determinantes de sedentarismo, tendo este por base de definição apenas as atividades de lazer. ${ }^{20}$ Outros autores ${ }^{7,10,11}$ descreveram uma relação próxima entre inatividade física, consumo de tabaco e outros aspectos que constituem um estilo de vida não saudável, constatando que estes tendem a agregar-se 
em alguns indivíduos. A atuação conjunta de fatores de risco resulta num aumento da morbilidade e redução na esperança média de vida.

Num estudo norte americano, ${ }^{15}$ tendo por base o Behavioral Risk Factor Surveillance System, observou-se que o consumo moderado de álcool se associa fortemente com maiores índices de atividade física, comparativamente com indivíduos abstémios. Abstémios reportaram ser $10 \%$ mais sedentários que os bebedores moderados. No presente estudo, o não consumo de bebidas alcoólicas associou-se em ambos os sexos e de forma significativa com maior intensidade de atividade física. No entanto, as estimativas calculadas podem estar subestimadas devido ao fato de os bebedores moderados se encontrarem na classe dos bebedores, devido à dificuldade de constituição de uma classe de bebedores moderados no Inquérito Nacional de Saúde.

O presente estudo possui algumas limitações, das quais destaca-se o fato de as variáveis terem sido auto-declaradas: demográficas, sociais e comportamentais, atividade física, peso e a altura. No entanto, o auto-relato de comportamentos e características é prática corrente nos inquéritos nacionais de saúde, sendo a forma mais viável de realizar medições em larga escala. Por outro lado, o largo tamanho amostral permite de alguma forma minimizar a imprecisão das estimativas calculadas.
Na presente análise para a definição do desfecho (ser ou não ativo) foi usada informação separada de três questões e não um conjunto de questões que constituíssem uma pontuação equivalente ao dispêndio energético. Esse tipo de questionário não possibilita boa discriminação, pelo fato de os indivíduos terem sido classificados em variáveis dependentes dicotômicas. Mesmo assim foi possível obter resultados significativos de acordo com pressupostos teóricos lógicos, como maior percentagem de indivíduos ativos no sexo masculino e mais jovens, o que confere alguma validade à classificação dos indivíduos.

A avaliação transversal limita o estabelecimento de associações sem equacionar a possibilidade de existir causalidade inversa, em particular para as variáveis comportamentais. Contudo, as diferenças sociais, demográficas e comportamentais podem explicar parcialmente a elevada prevalência de sedentarismo observada na população estudada, colocando Portugal como um dos países mais sedentários da União Européia.

\section{AGRADECIMENTOS}

Ao Observatório Nacional de Saúde (ONSA), Instituto Nacional de Saúde Dr. Ricardo Jorge, Ministério da Saúde de Portugal pela cessão da informação recolhida no Inquérito Nacional de Saúde 1998-99. 


\section{REFERÊNCIAS}

1. Audrain-McGovern J, Rodriguez D, Moss HB. Smoking progression and physical activity. Cancer Epidemiol Biomarkers Prev. 2003;12(11 Pt 1):1121-9.

2. Brownson RC, Boehmer TK, Luke DA. Declining rates of physical activity in the United States: what are the contributors? Annu Rev Public Health. 2005;26:42143.

3. Caspersen CJ, Powell KE, Christenson GM. Physical activity, exercise, and physical fitness: definitions and distinctions for health-related research. Public Health Rep. 1985;100(2):126-31.

4. Dunn AL, Trivedi MH, O'Neal HA. Physical activity dose-response effects on outcomes of depression and anxiety. Med Sci Sports Exerc. 2001;33(6Supl):S58797.

5. Elizondo-Armendariz JJ, Guillen Grima F, Aguinaga Ontoso I. Prevalencia de actividad física y su relación con variables sociodemográficas y estilos de vida en la población de 18 a 65 años de Pamplona. Rev Esp Salud Publica. 2005;79(5):559-67.

6. Erlichman J, Kerbey AL, James WP. Physical activity and its impact on health outcomes. Paper 1: The impact of physical activity on cardiovascular disease and all-cause mortality: an historical perspective. Obes Rev. 2002;3(4):257-71.

7. Ferrucci L, Izmirlian G, Leveille $S$, Phillips $C L$, Corti MC, Brock DB, et al. Smoking, physical activity, and active life expectancy. Am J Epidemiol. 1999;149(7):645-53

8. Gal DL, Santos AC, Barros H. Leisure-time versus full-day energy expenditure: a cross-sectional study of sedentarism in a Portuguese urban population. BMC Public Health. 2005;(5):16.

9. He XZ, Baker DW. Differences in leisure-time, household, and work-related physical activity by race, ethnicity, and education. J Gen Intern Med 2005;20(3):259-66.

10. Kaplan GA, Strawbridge WJ, Cohen RD, Hungerford LR. Natural history of leisure-time physical activity and its correlates: associations with mortality from all causes and cardiovascular disease over 28 years. Am J Epidemiol. 1996;144(8):793-7.
11. Kaplan MS, Newsom JT, McFarland BH, Lu L. Demographic and psychosocial correlates of physical activity in late life. Am J Prev Med. 2001;21(4):306-12.

12. Centers for Disease Control and Prevention (CDC). Trends in leisure-time physical inactivity by age, sex, and race/ethnicity-United States, 1994-2004. MMWR Morb Mortal Wkly Rep. 2005;54(39):991-4.

13. Marques-Vidal P, Dias CM. Trends in overweight and obesity in Portugal: the National Health Surveys 19956 and 1998-9. Obes Res. 2005;13(7):1141-5.

14. Monteiro CA, Conde WL, Matsudo SM, Matsudo VR, Bonseñor IM, Lotufo PA. A descriptive epidemiology of leisure-time physical activity in Brazil, 1996-1997. Rev Panam Salud Publica. 2003;14(4):246-54.

15. Mukamal KJ, Ding EL, Djoussé L. Alcohol consumption, physical activity, and chronic disease risk factors: a population-based cross-sectional survey. BMC Public Health. 2006;(6):118.

16. Parsons TJ, Power C, Manor O. Physical activity, television viewing and body mass index: a crosssectional analysis from childhood to adulthood in the 1958 British cohort. Int J Obes. 2005;29(10):1212-21.

17. Sallis JF. Age-related decline in physical activity: a synthesis of human and animal studies. Med Sci Sports Exerc. 2000;32(9):1598-600.

18. Schnohr P, Kristensen TS, Prescott E, Scharling H. Stress and life dissatisfaction are inversely associated with jogging and other types of physical activity in leisure time-The Copenhagen City Heart Study. Scand J Med Sci Sports. 2005;15(2):107-12.

19. Sherwood NE, Jeffery RW. The behavioral determinants of exercise: implications for physical activity interventions. Annu Rev Nutr. 2000;20:21-44.

20. Varo JJ, Martinez-Gonzalez MA, De Irala-Estévez J, Kearney J, Gibney M, Martínez JA. Distribution and determinants of sedentary lifestyles in the European Union. Int J Epidemiol. 2003;32(1):138-46.

21. Vaz de Almeida MD, Graca P, D'Amicis A, Lappalainen R, Damkjaer S. Physical activity levels and body weight in a nationally representative sample in the European Union. Public Health Nutr. 1999;2(1A):105-13. 